\title{
Major thrombo-embolic complications in abdominal and lower limb arteries in COVID19 patients: a case series.
}

\author{
giulia bonera ${ }^{1}$, giorgio nocivelli ${ }^{1}$, giorgio maria agazzi $^{1}$, raffaele cuomo ${ }^{1}$, and roberto \\ maroldi ${ }^{1}$ \\ ${ }^{1}$ Azienda Ospedaliera Spedali Civili di Brescia
}

April 29, 2020

\begin{abstract}
BACKGROUND: the outbreak of acute respiratory syndrome coronavirus 2 (SARS-CoV-2) has resulted in more than 150000 infected individuals and more than 20000 deaths in Italy. Viral acute pneumonitis is the major cause of morbidity and mortality, but there is increasing evidence that COVID-19 patients show a variable degree of vascular complications, due to a possible hypercoagulability status. OBJECTIVE: to report thrombotic events in abdominal and lower-limb arteries of COVID-19 patients (abdominal aorta, superior mesenteric artery, femoral arteries, cardiac, spleen and kidney). METHODS: we retrospectively reviewed our clinical records of patients with confirmed SARS-CoV-2 infection, admitted from March 1st to April 6th, 2020, to a tertiary care center in Brescia, Italy. We selected five patients with major arterial thrombo-embolic manifestations. RESULTS AND CONCLUSION: In our opinion, arterial thrombotic events may be more common in COVID-19 patients than in general population, possibly resulting in rapid worsening of patient conditions.
\end{abstract}

Keywords: covid-19; coronavirus; thrombosis; viral pneumonia; vascular complications.

\section{Abbreviations}

CT: computed tomography; ARDS: acute respiratory distress syndrome; ULN: upper limit of normality; CECT: contrast-enhanced computed tomography.

\section{BACKGROUND}

In late 2019 a novel form of coronavirus (SARS-CoV-2) was considered as the cause of a cluster of respiratory diseases in Wuhan, China. In late february 2020, after a rapid spread worldwide, the coronavirus disease (COVID-19) outbreak began in Italy, resulting up to now in more than 150000 infected individuals and more than 20000 deaths[1]. Despite the fact that viral acute pneumonitis is the major cause of morbidity and mortality, there is increasing evidence that patients with COVID-19 along with respiratory symptoms show a variable degree of systemic disease involvement[2]. Among those involving the cardiovascular system[3] there is evidence regarding a possible pathological hypercoagulability status, leading to higher reported embolic complications such as DIC or pulmonary embolism[4]. This prothrombotic state could be caused by the combined and synergic action of different factors related to covid-19: massive elevation of d-dimer[5], elevation of fibrinogen and inflammatory markers (the so called "cytokine storm"[6]), along with reported major thrombocytopenia[7] and indirect signs of endothelial disfunction (such as alterations in IL-6 and CRP levels[8]. Furthermore, COVID-19 positive patients could have also an intrinsic increased risk VTE due to prolonged immobilization following social distancing measures. This increased risk of potentially lethal thromboembolic complications, not only in the elderly with comorbidities but also in the younger age groups, led the major clinical societies (e.g. International Society of Thrombosis and Haemostasis) to suggest pharmacologic thromboprophylaxis to all hospitalized covid-19 patients, unless contraindicated[9]. However 
currently there are no recommendations for the outpatient setting, which involves a large number of patients (between $40-50 \%[1]$ ), and also data are lacking regarding arterial thrombotic risk in these patients.

\section{OBJECTIVE:}

Based on our clinical experience we report an unusual high rate of major thromboembolic events in abdominal and lower-limb arterial vessels in COVID-19 patients, showing a possible association between viral infection and extra-pulmonary thromboembolic disease.

\section{METHODS}

All cases came from Spedali Civili di Brescia, a tertiary care center with several dedicated COVID-19 units and a high number of COVID-19 patients (more than 10.000 in the province of Brescia), leading to a growing experience in the management of the coronavirus pandemic. All the included patients presented with typical COVID-19 clinical features (fever, fatigue, dry cough or dyspnea) with either a positive SARS-CoV-2 nasopharyngeal swab or a high resolution lung CT highly suggestive of viral pneumonia.

\section{RESULTS AND DISCUSSION}

We report a case series of five patients with confirmed SARS-CoV-2 infection and major arterial thromboembolic complications. Patient's characteristics are resumed in Table 1.

\section{PATIENT 1}

A 61-year old male presented to the emergency room complaining fever and dyspnea; his SARS-CoV-2 nasopharyngeal swab was positive and chest x-ray demonstrated smooth airspace opacities in the lower left lung. Six days after the admission the patient reported acute lower limbs pain with no femoral pulses. CECT showed a complete occlusion of the infrarenal abdominal aorta (Figure 1), with thrombus extending into the common and external iliac arteries and into the common femoral arteries requiring urgent bilateral surgical thrombectomy; anticoagulation therapy with heparin was started. Thoracic CT findings were typical for COVID-19, with bilateral peripheral ground glass opacities, crazy-paving appearance, lack of pleural effusion. Nine days after the admission (three after surgery), the patient developed lower back pain associated with haemoglobin drop and the CT demonstrated bilateral lumbar hematoma, treated with endovascular embolization.

\section{PATIENT 2}

A 60-year old male was diagnosed of COVID-19 (SARS-CoV-2 positive nasopharyngeal swab) after a week of fever, cough and shortness of breath. Mechanical ventilation was needed. Fifteen days later because of the worsening respiratory status the patient underwent CT that showed typical COVID-19 pulmonary findings and right pulmonary embolism. In the following days there was a worsening of the respiratory status with development of ARDS. One month after his admission the patient developed acute mesenteric ischemia: CT showed non-occlusive thrombus in the proximal superior mesenteric artery (Figure 2) and endoluminal irregularities of the middle colic artery.

\section{PATIENT 3}

A 71-year old patient was transferred to our institution for cerebral vascular ischemia. During the hospitalization was diagnosed of COVID-19 on the basis of typical clinical and chest x-ray findings (nasopharyngeal swabs were negative). Two days after his admission a surge in D-dimer levels was noted $(>2200 \mathrm{ng} / \mathrm{ml})$ and, to rule out pulmonary embolism, a CT was performed: imaging was negative for pulmonary embolism but demonstrated a hypodense lesion in the left ventricle indicative of thrombus (confirmed echocardiographically) and a hypodense, infarctual area in the splenic parenchyma (Figure 3). These findings were not detectable in a previous follow-up CT performed in January 2020.

\section{PATIENT 4}


A 71-year old male patient with a history of lymphoma presented to the emergency department for fever and dyspnea. Chest x-ray was typical of COVID-19. Eleven days after the admission, the patient underwent ct scan for worsening lymphocytopenia: massive ground-glass opacifications were noted in both lungs. a hypodense parenchymal wedge-shaped lesion, highly suggestive of infarction, was recognizable in the left kidney. this finding was not detectable in a previous ct scan performed one month before the onset of respiratory symptoms.

\section{PATIENT 5}

A 85-year old man reporting persistent fever, history of contact with a laboratory-confirmed COVID-19 case and sudden onset of left lower limb pain presented to the emergency department. SARS-CoV-2 infection was confirmed by a positive nasopharyngeal swab and by typical findings in the chest ct scan. doppler ultrasound examination demontrated a long occlusion of the superficial femoral artery, treated with surgical thrombectomy and subsequent angioplasties of some underlying arterial stenosis.

The purpose of the current paper is to report a possible association between the novel SARS-CoV-2 infection and a higher rate of major arterial thrombo-embolic events. The clinical characteristics of the included subjects are in keeping with current published literature, as we noted an unfavourable prognosis mostly in older male patients with higher level of pro-inflammatory factors and d-dimer (sometimes $>10 \mathrm{ULN}$ ), as recent studies outlined[5,8,10-12]. In particular older age and coagulation disfunctions were related to ARDS development and subsequent death afterwards[8]. The higher incidence of arterial events we observed could be related to the loss of equilibrium between prothrombotic and antithrombotic factors during severe pulmonary infections. Arterial thrombosis has a different pathogenesis compared to venous thromboembolism and is related to endothelial disfunction and endovascular damage. The majority of patients with COVID-19 are reported to have cardiovascular comorbidities such as hypertension and diabetes[8] which are known to be associated with endothelial disfunction[13,14], and when present these comorbidities are associated with a worse prognosis[8]. Pulmonary infections are knows to increase the risk of thrombo-embolic diseases with peak observed during active disease or shortly afterwards[15]. Major thrombo-embolic events have been reported following viral infections in past pandemic outbreaks[16,17]), with high as $25 \%$ prevalence in patients with severe manifestations of H1N1 influenza[18]. However further studies are needed addressing the link between thrombo-embolism and COVID-19 because current published data are sparse[19].

In our opinion, arterial thrombotic events may be more common in COVID-19 patients than is generally appreciated. The clinical impact is not negligible since a major thrombo-embolic event could be unexpected and result in rapid worsening of patient conditions, ultimately leading to death. Clinicians should be more aware of this uncommonly reported complications and have lower thresholds compared to routine clinical practice for the identification of high risk patients even in the outpatient setting, for starting appropriate thrombotic prophylaxis[20] and prescribing further diagnostic evaluation or blood test[21] in COVID-19 patients.

\section{REFERENCES}

1 GIMBE Evidence for Health: Covid monitoring.

2 Driggin E, Madhavan MV, Bikdeli B, Chuich T, Laracy J, Bondi-Zoccai G, Brown TS, Nigoghossian CD, Zidar DA, Haythe J, Brodie D, Beckman JA, Kirtane AJ, Stone GW, Krumholz HM, Parikh SA. Cardiovascular Considerations for Patients, Health Care Workers, and Health Systems During the Coronavirus Disease 2019 (COVID-19) Pandemic. J Am Coll Cardiol 2020; .

3 Inciardi RM, Lupi L, Zaccone G, Italia L, Raffo M, Tomasoni D, Cani DS, Cerini M, Farina D, Gavazzi E, Maroldi R, Adamo M, Ammirati E, Sinagra G, Lombardi CM, Metra M. Cardiac Involvement in a Patient With Coronavirus Disease 2019 (COVID-19). JAMA Cardiol 2020; .

4 Madjid M, Safavi-Naeini P, Solomon SD, Vardeny O. Potential Effects of Coronaviruses on the Cardiovascular System: A Review. JAMA Cardiol 2020; . 
5 Han H, Yang L, Liu R, Liu F, Wu K, Li J, Liu X, Zhu C. Prominent changes in blood coagulation of patients with SARS-CoV-2 infection. Clinical Chemistry and Laboratory Medicine (CCLM) 2020;0 .

$6 \mathrm{Li} \mathrm{T}, \mathrm{Lu} \mathrm{H}$, Zhang W. Clinical observation and management of COVID-19 patients. Emerg Microbes Infect 2020; $9: 687-90$.

7 Lippi G, Plebani M, Henry BM. Thrombocytopenia is associated with severe coronavirus disease 2019 (COVID-19) infections: A meta-analysis. Clin Chim Acta 2020; 506 : 145-8.

8 Wu C, Chen X, Cai Y, Xia J, Zhou X, Xu S, Huang H, Zhang L, Zhou X, Du C, Zhang Y, Song J, Wang S, Chao Y, Yang Z, Xu J, Zhou X, Chen D, Xiong W, Xu L, et al. Risk Factors Associated With Acute Respiratory Distress Syndrome and Death in Patients With Coronavirus Disease 2019 Pneumonia in Wuhan, China. JAMA Intern Med 2020; .

9 George L Anesi et al. Coronavirus disease 2019 (COVID-19): Critical care issues. UpToDate;

10 Tang N, Li D, Wang X, Sun Z. Abnormal coagulation parameters are associated with poor prognosis in patients with novel coronavirus pneumonia. J Thromb Haemost 2020; 18 : 844-7.

11 Lillicrap D. Disseminated intravascular coagulation in patients with 2019-nCoV pneumonia. J Thromb Haemost 2020; 18 : 786-7.

12 Lippi G, Favaloro EJ. D-dimer is Associated with Severity of Coronavirus Disease 2019: A Pooled Analysis. Thromb Haemost 2020; : s-0040-1709650.

13 Feinbloom D, Bauer KA. Assessment of Hemostatic Risk Factors in Predicting Arterial Thrombotic Events. ATVB 2005; 25 : 2043-53.

14 Pechlivani N, Ajjan RA. Thrombosis and Vascular Inflammation in Diabetes: Mechanisms and Potential Therapeutic Targets. Front Cardiovasc Med 2018; 5 : 1.

15 Beristain-Covarrubias N, Perez-Toledo M, Thomas MR, Henderson IR, Watson SP, Cunningham AF. Understanding Infection-Induced Thrombosis: Lessons Learned From Animal Models. Front Immunol 2019;10 : 2569 .

16 Bunce PE, High SM, Nadjafi M, Stanley K, Liles WC, Christian MD. Pandemic H1N1 Influenza Infection and Vascular Thrombosis. Clinical Infectious Diseases 2011; 52 : e14-7.

17 Hüzmeli C, Saglam M, Arıkan A, Doner B, Akıncı G, Candan F. Infrarenal Aorta Thrombosis Associated with H1N1 Influenza A Virus Infection. Case Reports in Infectious Diseases 2016;2016 : 1-3.

18 Avnon LS, Munteanu D, Smoliakov A, Jotkowitz A, Barski L. Thromboembolic events in patients with severe pandemic influenza A/H1N1.European Journal of Internal Medicine 2015; 26 : 596-8.

19 Danzi GB, Loffi M, Galeazzi G, Gherbesi E. Acute pulmonary embolism and COVID-19 pneumonia: a random association? European Heart Journal 2020; : ehaa254.

20 Tang N, Bai H, Chen X, Gong J, Li D, Sun Z. Anticoagulant treatment is associated with decreased mortality in severe coronavirus disease 2019 patients with coagulopathy. J Thromb Haemost 2020; .

21 Favaloro EJ, Lippi G. Recommendations for Minimal Laboratory Testing Panels in Patients with COVID19: Potential for Prognostic Monitoring.Semin Thromb Hemost 2020; : s-0040-1709498.

\section{ACKNOWLEDGMENTS}

G.B.,G.N.,G.M.A.: concept and design, critical writing

R.C, R.M.: critical writing, revision, final approval

CONFLICT OF INTEREST STATEMENT

The Authors declare no conflict of interests 


\section{FIGURES:}

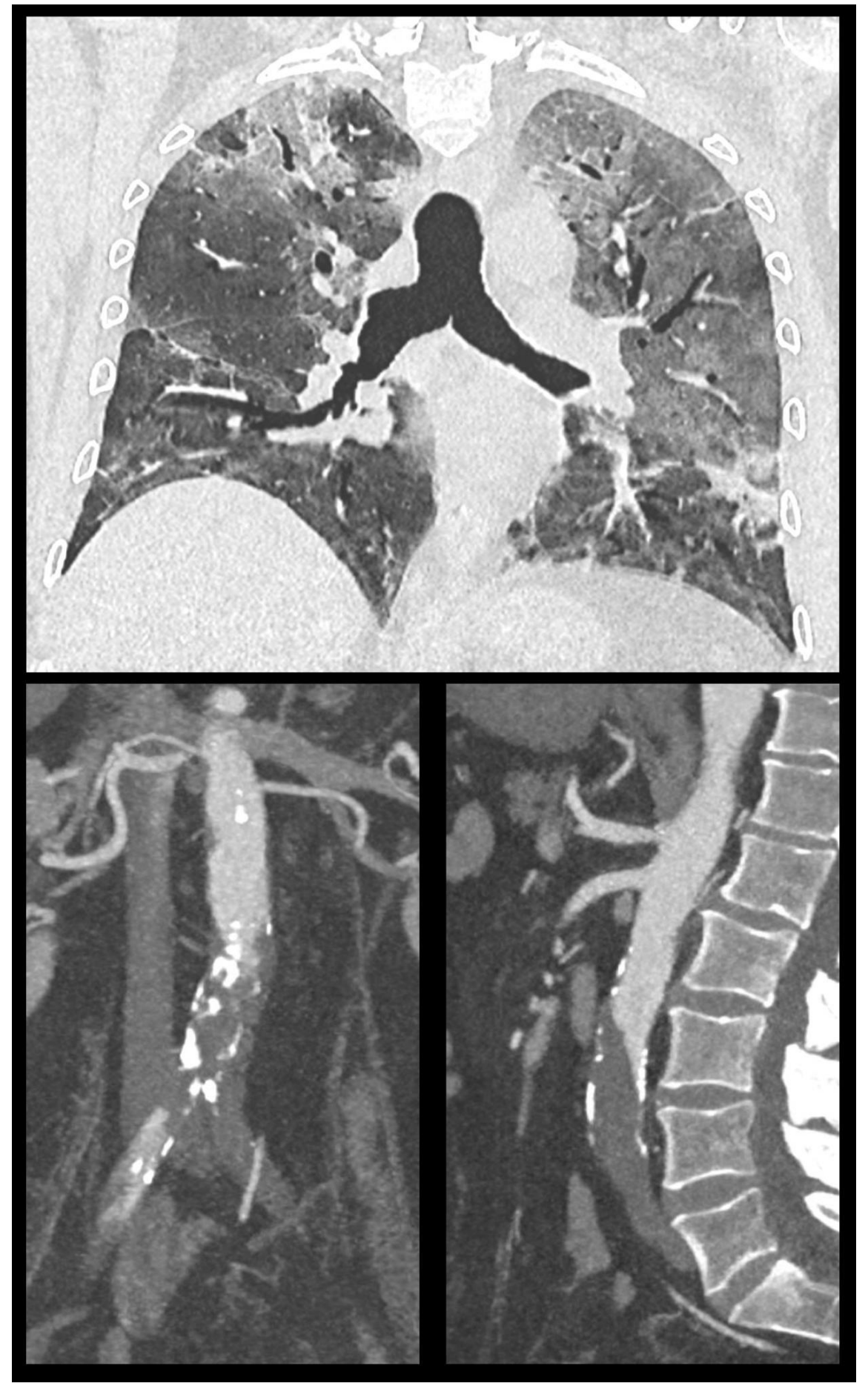

Figure 1: CECT in Patient 1 showing extensive ground-glass opacities in both lungs (A) typical for COVID19. Acute thrombotic occlusion of the infrarenal abdominal aorta extending to the iliac arteries $(\mathrm{B}, \mathrm{C})$. 


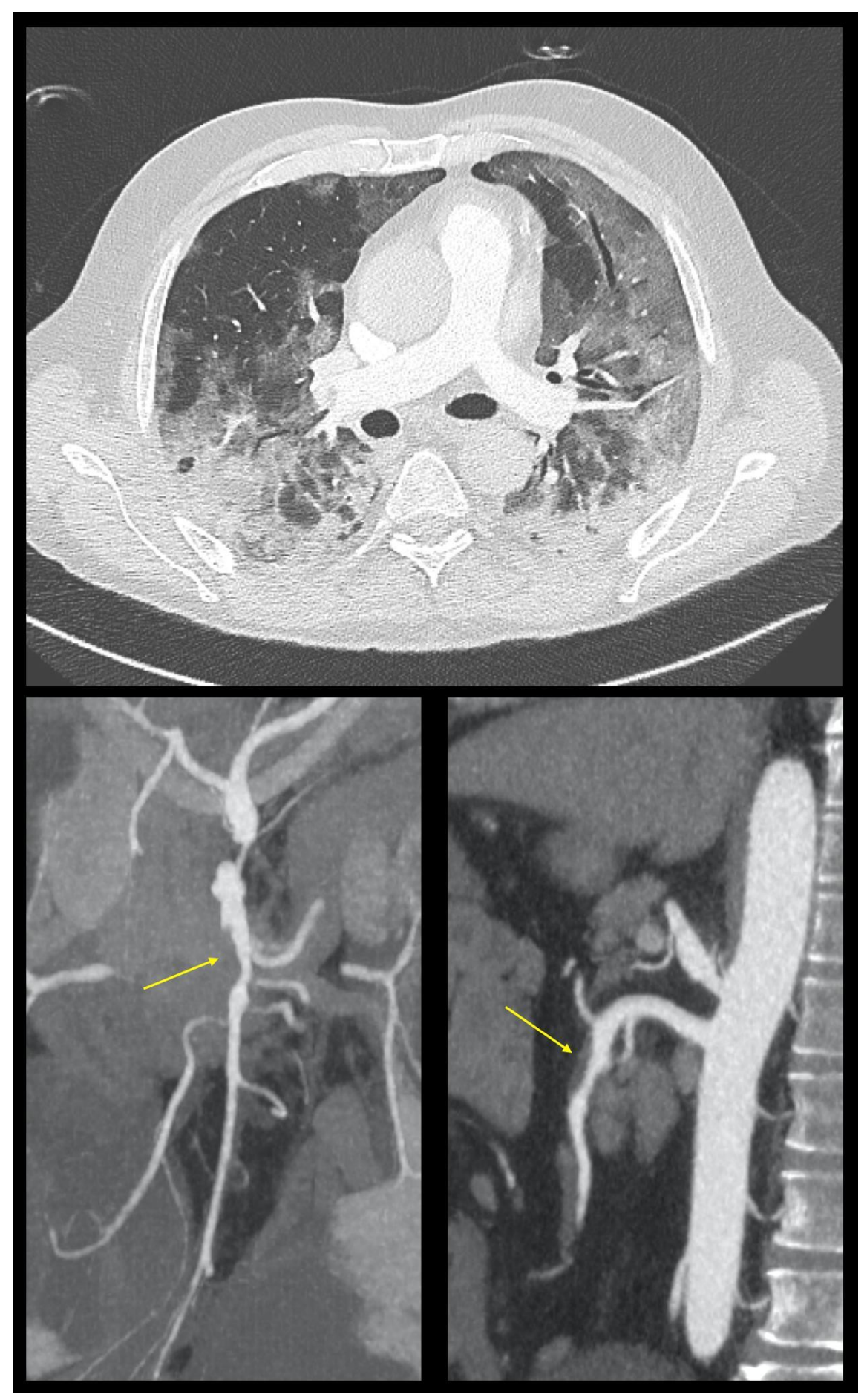

Figure 2: CECT of Patient 2 showing bilateral ground-glass opacities (A); non-occlusive thrombus in the proximal superior mesenteric artery in B and C (coronal and sagittal reconstructions, arrows). 


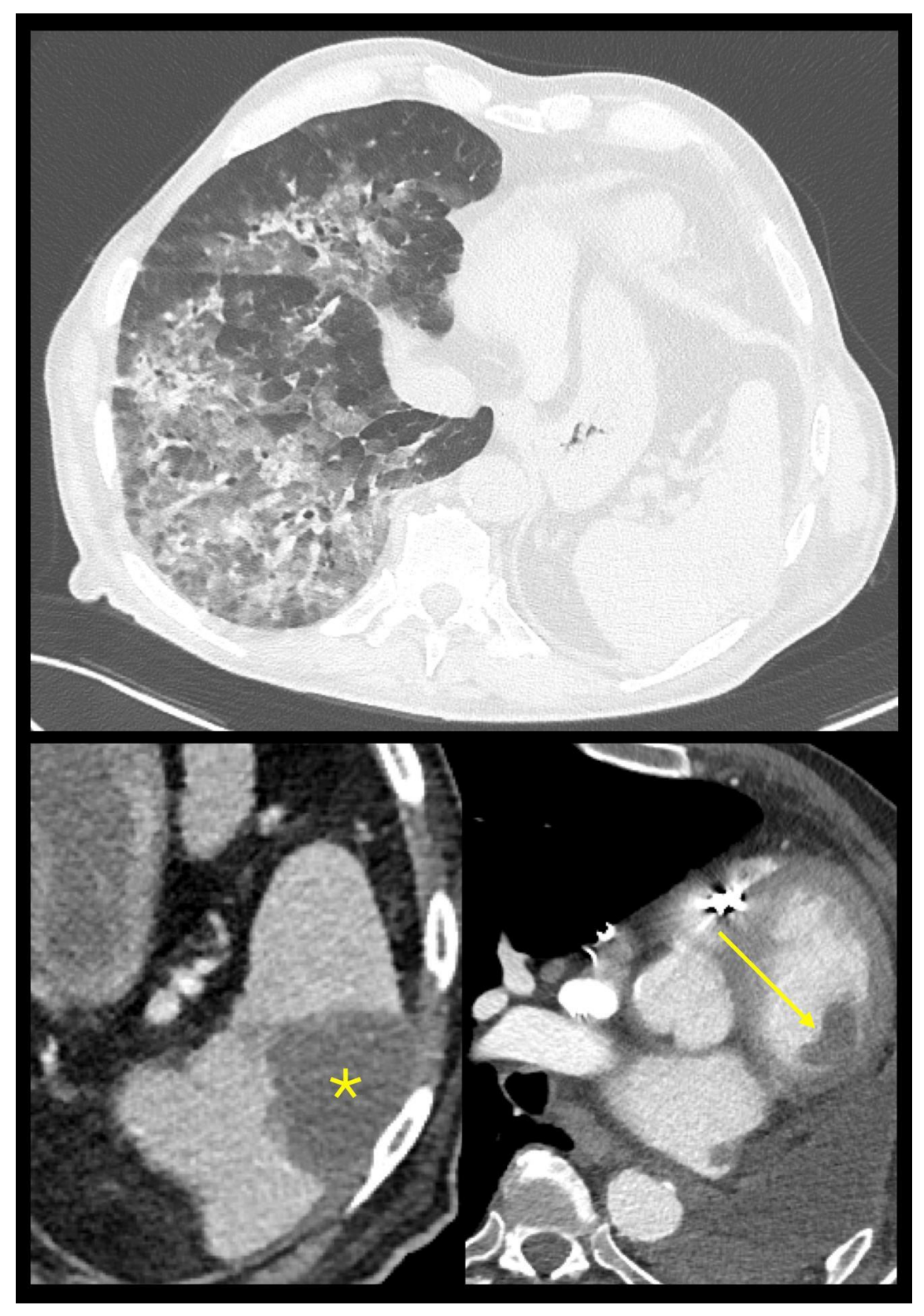

Figure 3: CECT of Patient 3 demonstrating ground-glass opacities and crazy-paving pattern in the right lung (A). In B, infarctual intraparenchymal splenic lesion $\left(^{*}\right)$; in $\mathrm{C}$, perfusion defect in the left ventricle in keeping with thrombus (arrow).

\section{Hosted file}

Table file.docx available at https://authorea.com/users/316441/articles/446590-major-thromboembolic-complications-in-abdominal-and-lower-limb-arteries-in-covid19-patients-a-case-series 


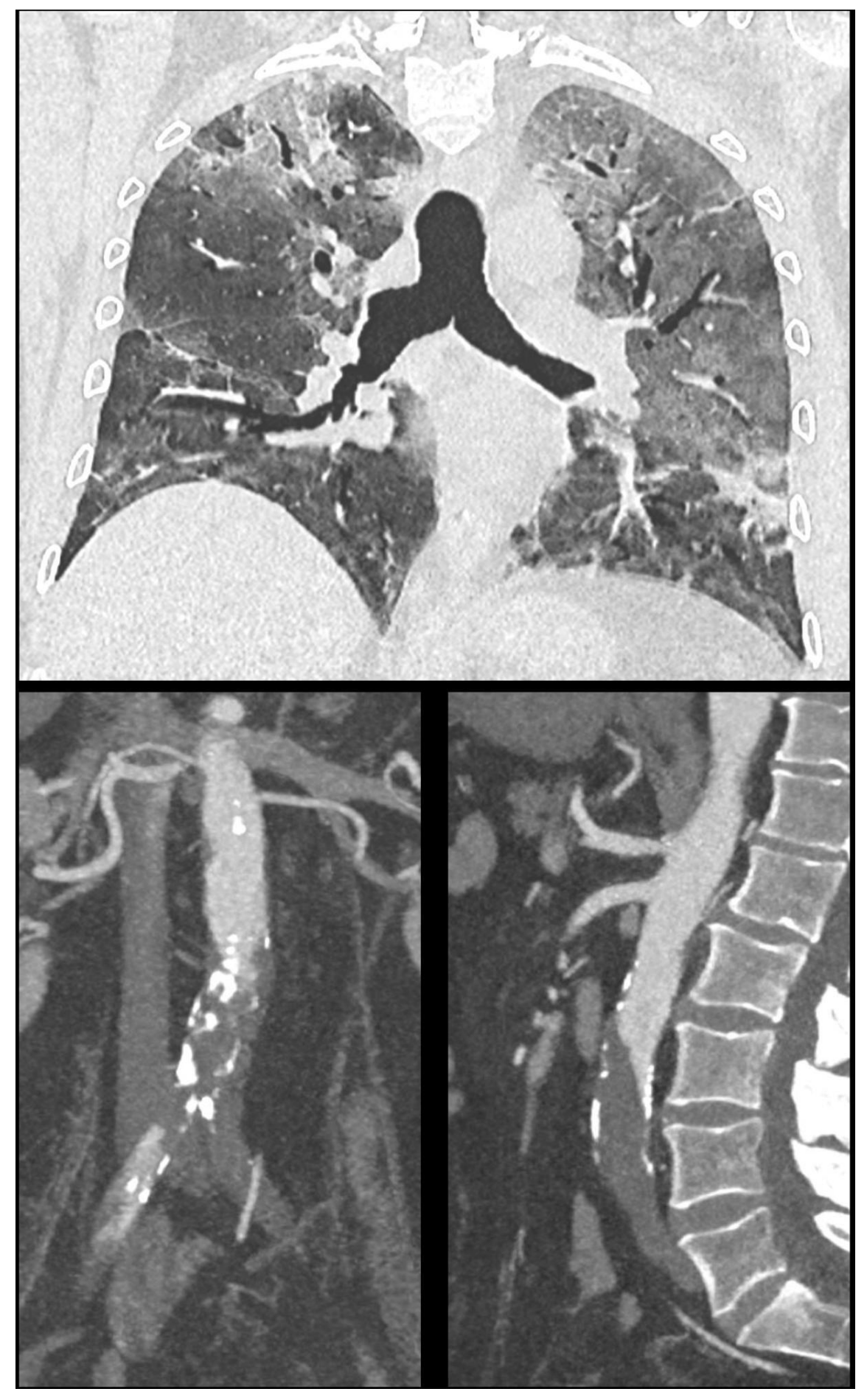




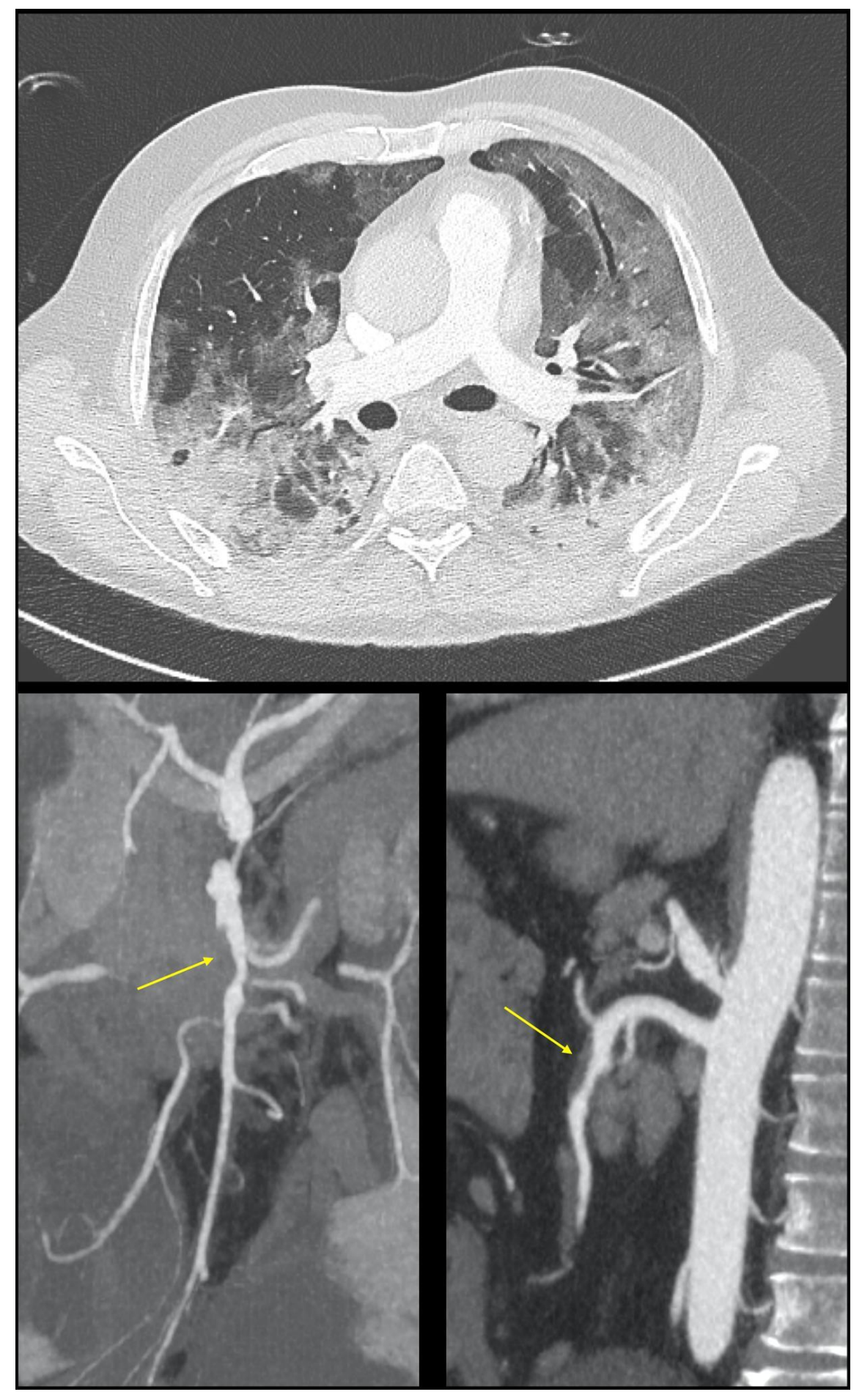




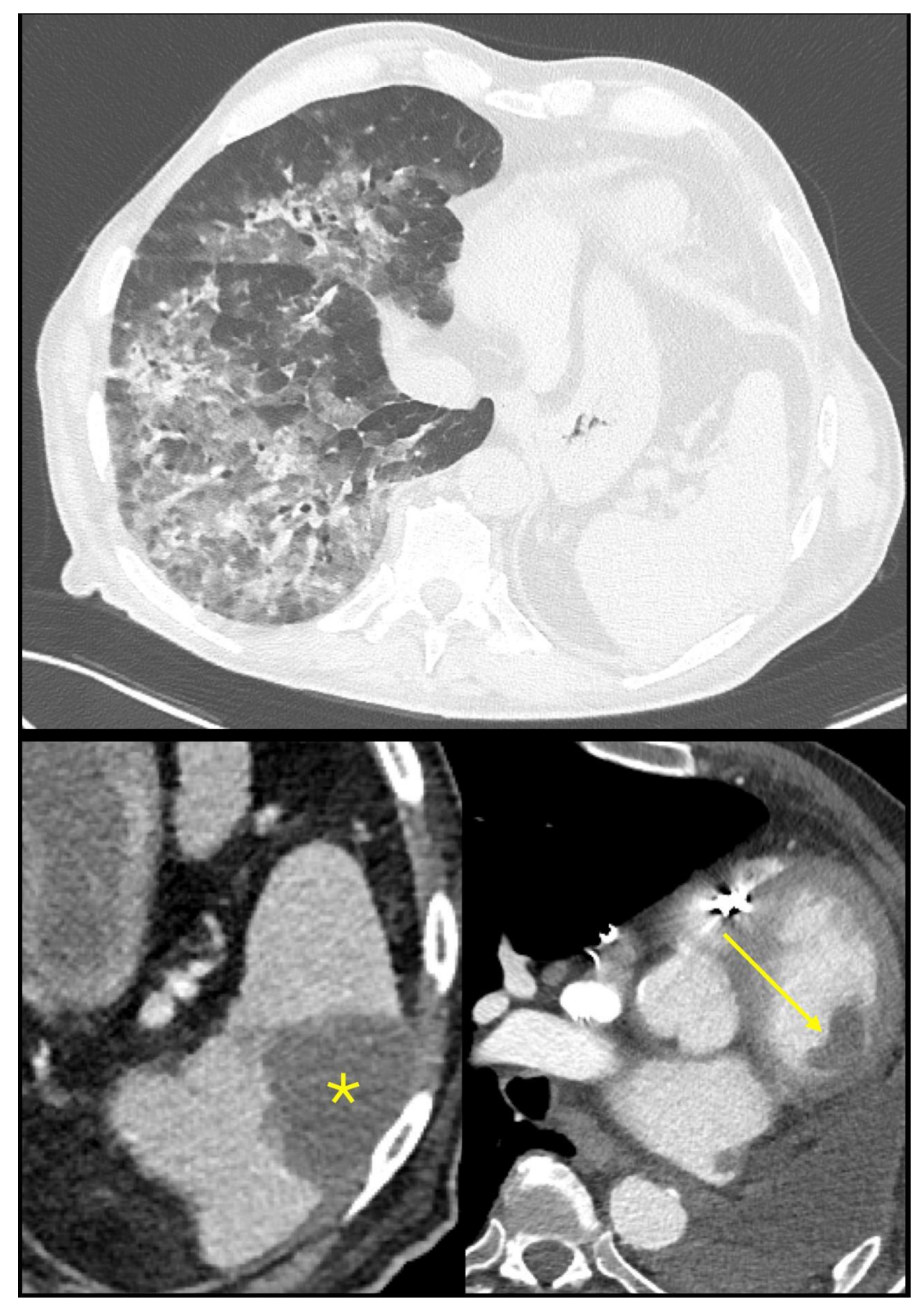

